\title{
THE WU METRIC IS NOT UPPER SEMICONTINUOUS
}

\author{
PIOTR JUCHA
}

(Communicated by Mei-Chi Shaw)

\begin{abstract}
We give an example of a bounded pseudoconvex domain in $\mathbb{C}^{n}$, the $\mathrm{Wu}$ metric of which (associated to the Kobayashi-Royden or the Azukawa metric) is not upper semicontinuous.
\end{abstract}

$\mathrm{H}$. Wu defined in $[\mathrm{Wu} 2]$ an invariant metric which was to combine invariant properties of the Kobayashi-Royden metric and regularity properties of Kähler metrics. The metric, called later the $\mathrm{Wu}$ metric, was studied in several papers (e.g. [Che-Kim 1], [Che-Kim 2], [Juc], [Jar-Pfl 3], [Jar-Pfl 2]). Jarnicki and Pflug pointed out ([Jar-Pfl 3], [Jar-Pfl 2]) that such an elementary property as its upper semicontinuity had not been completely understood. It was claimed ([Wu 2], [Wu 1]) or cited ([Che-Kim 1], [Juc]) without proof that the Wu metric is an upper semicontinuous pseudo-Hermitian metric defined on every complex manifold. We are going to present an example of a bounded pseudoconvex domain in $\mathbb{C}^{n}$ which contradicts that statement.

\section{Definition}

We present the sketch of the definition of the $\mathrm{Wu}$ metric in an abstract setting ([Jar-Pfl 3]). For detailed discussion we refer the reader to [Jar-Pfl 3] (or [Jar-Pfl 2]) and [Wu 2].

Let $p: \mathbb{C}^{n} \rightarrow \mathbb{R}_{+}$be a $\mathbb{C}$-seminorm. Put:

$$
\begin{aligned}
I(p):= & \left\{X \in \mathbb{C}^{n}: p(X)<1\right\}, \\
V(p):= & \left\{X \in \mathbb{C}^{n}: p(X)=0\right\}, \\
U(p):= & \text { the orthogonal complement of } V \text { with respect to } \\
& \text { the standard scalar product in } \mathbb{C}^{n} ; \\
I_{0}(p):= & I(p) \cap U(p) .
\end{aligned}
$$

Notice that $I_{0}(p)$ is bounded.

For any pseudo-Hermitian scalar product $s: \mathbb{C}^{n} \times \mathbb{C}^{n} \rightarrow \mathbb{C}$, define

$$
q_{s}(X):=\sqrt{s(X, X)}, \quad X \in \mathbb{C}^{n} .
$$

Received by the editors October 13, 2006 and, in revised form, February 12, 2007.

2000 Mathematics Subject Classification. Primary 32F45.

Key words and phrases. Wu metric, invariant metrics.

This work was supported by Research Grant No. 1 PO3A 00528 of the Polish Ministry of Science and Higher Education. 
Let $\mathcal{F}(p)$ be the set of all pseudo-Hermitian scalar products $s: \mathbb{C}^{n} \times \mathbb{C}^{n} \rightarrow \mathbb{C}$ such that $q_{s} \leq p$ (or, equivalently, $I(p) \subset I\left(q_{s}\right)$ ). There exists a unique (!) element $s(p) \in \mathcal{F}(p)$ which is maximal with respect to the partial ordering $\prec$ defined for $a, b \in \mathcal{F}(p)$ :

$$
a \prec b \text { if } \operatorname{det}\left[a\left(e_{j}, e_{k}\right)\right]_{j, k=1, \ldots, m} \leq \operatorname{det}\left[b\left(e_{j}, e_{k}\right)\right]_{j, k=1, \ldots, m},
$$

for any basis $\left(e_{1}, \ldots, e_{m}\right)$ of $U(p)$. In other words, $I_{0}\left(q_{s(p)}\right)$ is an ellipsoid that has minimal volume of all ellipsoids containing the $p$-ball $I_{0}(p)$. This ellipsoid is also called (cf. [Che-Kim 1], [Che-Kim 2]) "the best fitting ellipsoid".

We define

$$
\begin{aligned}
& \widetilde{\mathbb{W}} p(X):=q_{s(p)}(X), \quad X \in \mathbb{C}^{n} ; \\
& \mathbb{W} p(X):=\sqrt{m} \widetilde{\mathbb{W}} p(X), \quad X \in \mathbb{C}^{n},
\end{aligned}
$$

where $m:=\operatorname{dim} U(p)$.

For a domain $D \subset \mathbb{C}^{n}$, denote by $\mathcal{M}(D)$ the space of all pseudometrics

$$
\eta: D \times \mathbb{C}^{n} \rightarrow \mathbb{R}_{+}, \quad \eta(a ; \lambda X)=|\lambda| \eta(a ; X), \quad \lambda \in \mathbb{C},(a, X) \in D \times \mathbb{C}^{n},
$$

such that

$$
\forall a \in D \exists M, r>0: \eta(z ; X) \leq M\|X\|, \quad z \in \mathbb{B}(a, r) \subset D, X \in \mathbb{C}^{n},
$$

where $\mathbb{B}(a, r):=\left\{z \in \mathbb{C}^{n}:\|z-a\|<r\right\}$ and $\|\cdot\|$ is the standard Euclidean norm.

For $\eta \in \mathcal{M}(D)$ we define the $\mathrm{Wu}$ pseudometric:

$$
(\mathbb{W} \eta)(a ; X):=(\mathbb{W} \widehat{\eta}(a ; \cdot))(X), \quad a \in D, X \in \mathbb{C}^{n} .
$$

Here $\widehat{\eta}$ denotes the Busemann pseudometric associated to $\eta$ (cf. e.g. [Jar-Pfl 1]), i.e.

$$
\widehat{\eta}(a ; X):=\sup \{p(X)\}, \quad a \in D, X \in \mathbb{C}^{n},
$$

where the supremum is taken over all $\mathbb{C}$-seminorms $p$ such that $p \leq \eta(a ; \cdot)$.

For convenience we define also

$$
(\widetilde{\mathbb{W}} \eta)(a ; X):=(\widetilde{\mathbb{W}} \widehat{\eta}(a ; \cdot))(X), \quad a \in D, X \in \mathbb{C}^{n} .
$$

Some basic properties of the $\mathrm{Wu}$ pseudometric are listed in Proposition 1.

We denote by $\Delta$ the open unit disk in $\mathbb{C}$. Let $\left(\eta_{D}\right)_{D}$ be a family of pseudometrics defined for all domains $D \subset \mathbb{C}^{n}, n \geq 1$. We call $\left(\eta_{D}\right)_{D}$ a holomorphically contractible family of pseudometrics (cf. [Jar-Pfl 1], [Jar-Pfl 2]) if we have

$$
\begin{gathered}
\eta_{\Delta}(z ; X)=\frac{|X|}{1-|z|^{2}}, \quad z \in \Delta, X \in \mathbb{C}, \\
\eta_{D_{2}}\left(F(z) ; F^{\prime}(z) X\right) \leq \eta_{D_{1}}(z ; X), \quad z \in D_{1}, X \in \mathbb{C}^{n_{1}},
\end{gathered}
$$

for domains $D_{1} \subset \mathbb{C}^{n_{1}}, D_{2} \subset \mathbb{C}^{n_{2}}$ and every holomorphic mapping $F: D_{1} \rightarrow D_{2}$.

We say that the family $\left(\eta_{D}\right)_{D}$ has the product property if

$$
\begin{aligned}
& \eta_{D_{1} \times D_{2}}((z, w) ;(X, Y))=\max \left(\eta_{D_{1}}(z ; X), \eta_{D_{2}}(w ; Y)\right), \\
& (z, w) \in D_{1} \times D_{2},(X, Y) \in \mathbb{C}^{n_{1}} \times \mathbb{C}^{n_{2}}
\end{aligned}
$$

for any domains $D_{1} \subset \mathbb{C}^{n_{1}}, D_{2} \subset \mathbb{C}^{n_{2}}, n_{1}, n_{2} \geq 1$.

Proposition 1 (cf. [Wu 2], [Jar-Pfl 3], [Jar-Pfl 2]).

(a) If $\eta \in \mathcal{M}(D)$ is a continuous metric, then so is $\mathbb{W} \eta$. 
(b) If $\left(\eta_{D}\right)_{D}$ is a holomorphically contractible family of pseudometrics, then for any biholomorphic mapping $F: D_{1} \rightarrow D_{2}\left(D_{j} \subset \mathbb{C}^{n}, j=1,2\right)$ we have

$$
\mathbb{W} \eta_{D_{2}}\left(F(z) ; F^{\prime}(z) X\right)=\mathbb{W} \eta_{D_{1}}(z ; X), \quad z \in D_{1}, X \in \mathbb{C}^{n} .
$$

(c) If $\left(\eta_{D}\right)_{D}$ is a holomorphically contractible family of pseudometrics, then for any holomorphic mapping $F: D_{1} \rightarrow D_{2}\left(D_{1} \subset \mathbb{C}^{n_{1}}, D_{2} \subset \mathbb{C}^{n_{2}}\right)$ we have

$$
\mathbb{W} \eta_{D_{2}}\left(F(z) ; F^{\prime}(z) X\right) \leq \sqrt{n_{2}} \mathbb{W} \eta_{D_{1}}(z ; X), \quad z \in D_{1}, X \in \mathbb{C}^{n} .
$$

(d) If $\left(\eta_{D}\right)_{D}$ is a family of pseudometrics satisfying the product property, then

$$
\begin{aligned}
\mathbb{W} \eta_{D_{1} \times D_{2}}((z, w) ;(X, Y)) & =\left(\left(\mathbb{W} \eta_{D_{1}}(z ; X)\right)^{2}+\left(\mathbb{W} \eta_{D_{2}}(w ; Y)\right)^{2}\right)^{\frac{1}{2}} \\
(z, w) & \in D_{1} \times D_{2} \subset \mathbb{C}^{n_{1}} \times \mathbb{C}^{n_{1}},(X, Y) \in \mathbb{C}^{n_{1}} \times \in \mathbb{C}^{n_{2}} .
\end{aligned}
$$

Recall definitions of the $k$-th Carathéodory-Reiffen $\left(\gamma^{(k)}\right)$, Azukawa $(A)$ and Kobayashi-Royden $(\kappa)$ pseudometrics. For details and properties see for example [Jar-Pfl 1], [Jar-Pfl 2].

For a domain $D \subset \mathbb{C}^{n}$ and $a \in D, X \in \mathbb{C}^{n}, k \in \mathbb{N} \backslash\{0\}$ define

$$
\begin{array}{r}
\gamma_{D}^{(k)}(a ; X):=\sup \left\{\left|\frac{1}{k !} f^{(k)}(a) X\right|^{\frac{1}{k}}: f \in \mathcal{O}(D, \Delta), \operatorname{ord}_{a} f \geq k\right\} ; \\
A_{D}(a ; X):=\sup \left\{\limsup _{0 \neq \lambda \rightarrow 0} \frac{v(a+\lambda X)}{|\lambda|}: v: D \rightarrow[0,1)\right. \text { is log-psh, } \\
\exists M, r>0: v(z) \leq M\|z-a\| \text { if }\|z-a\|<r\} ; \\
\kappa_{D}(a ; X):=\inf \left\{t>0: \exists \varphi \in \mathcal{O}(\Delta, D): \varphi(0)=a, t \varphi^{\prime}(0)=X\right\} .
\end{array}
$$

\section{Problem}

Jarnicki and Pflug pointed out that the continuity of $\mathbb{W} \eta$ could be proved only under the assumption that $\eta$ is continuous. The question of the upper semicontinuity of the $\mathrm{Wu}$ metric appears in a natural way, for instance in the definition of the integrated form $\int(\mathbb{W} \eta)$. In general, the upper semicontinuity of $\eta$ does not imply the upper semicontinuity of $\mathbb{W} \eta$ (cf. [Jar-Pfl 3], [Jar-Pfl 2]). In the case where $\eta$ is the Kobayashi-Royden (pseudo)metric the problem has remained open, even though $\mathrm{Wu}$ (cf. [Wu 1], [Wu 2]) and Cheung and Kim (cf. [Che-Kim 1]) claimed (without proof) the upper semicontinuity of $\mathbb{W} \kappa_{D}$.

Jarnicki and Pflug ([Jar-Pfl 3], [Jar-Pfl 2]) stated the following question:

$$
\text { Let } \alpha \in\left\{\gamma_{D}^{(k)}, A_{D}, \kappa_{D}\right\} \text {. Is } \mathbb{W} \alpha \text { upper semicontinuous? }
$$

We construct a bounded pseudoconvex domain $G \subset \mathbb{C}^{n}$ such that $\mathbb{W} \kappa_{G}$ and $\mathbb{W} A_{G}$ are not upper semicontinuous. We get even more: if $\left(\alpha_{D}\right)_{D}$ is a holomorphically contractible family of metrics such that $\alpha_{D} \in \mathcal{M}(D)$ and $A_{D} \leq \alpha_{D} \leq \kappa_{D}$ for any domain $D \subset \mathbb{C}^{n}$, then $\mathbb{W} \alpha_{G}$ is not upper semicontinuous.

In view of Proposition $1, \mathbb{W} \gamma_{D}^{(k)}$ is a continuous metric for a bounded domain $D$, since $\gamma_{D}^{(k)}$ is a continuous metric (cf. [Jar-Pfl 1]).

The problem remains open for $\gamma^{(k)}$ in the class of domains that are not biholomorphic to any bounded domain. 


\section{Counterexample}

To simplify the notation let $\mathbb{B}_{\eta_{D}}(a):=I\left(\eta_{D}(a ; \cdot)\right)$ denote the unit $\eta_{D}$-ball centered at a point $a \in D\left(\right.$ for $\left.\eta_{D} \in \mathcal{M}(D)\right)$ and let $\mathcal{E}(B):=I\left(\widetilde{\mathbb{W}} \widehat{h}_{B}\right)$ stand for the smallest ellipsoid containing the balanced domain $B$. Here, $h_{B}$ is the Minkowski functional of the domain $B$.

Remark 2. The construction of the Wu metric determines that

(a) there exists a unique $\mathcal{E}(B)$ for any balanced domain $B \subset \mathbb{C}^{n}$;

(b) if $p$ is a $\mathbb{C}$-seminorm, then $\widetilde{\mathbb{W}} p=h_{\mathcal{E}(I(p))}$. Consequently, finding $\mathcal{E}\left(\mathbb{B}_{\eta_{D}}(a)\right)$ is equivalent to finding $\widetilde{\mathbb{W}} \eta_{D}(a ; \cdot)$ and $\mathbb{W} \eta_{D}(a ; \cdot)$ for $\eta_{D} \in \mathcal{M}(D), D \subset \mathbb{C}^{n}$, $a \in D$.

Remark 3. If $B$ is a balanced Reinhardt domain, then

(a) $\mathcal{E}(B)$ is a complete Reinhardt domain;

(b) there exist $a_{1}, \ldots, a_{n} \geq 0$ such that

$$
\widetilde{\mathbb{W}} \widehat{h}_{B}(X)=\left(s\left(\widehat{h}_{B}\right)(X, X)\right)^{\frac{1}{2}}=h_{\mathcal{E}(B)}(X)=\left(\sum_{j=1}^{n} a_{j}\left|X_{j}\right|^{2}\right)^{\frac{1}{2}}, \quad X \in \mathbb{C}^{n} .
$$

Proof of Remark 3. The set $B$ is invariant under the action of

$$
\Phi_{\Lambda}(X):=\left(\lambda_{1} X_{1}, \ldots, \lambda_{n} X_{n}\right), \quad X \in \mathbb{C}^{n},
$$

where $\Lambda=\left(\lambda_{1}, \ldots, \lambda_{n}\right) \in(\partial \Delta)^{n}$. Thus $\Phi_{\Lambda}(\mathcal{E}(B))=\mathcal{E}\left(\Phi_{\Lambda}(B)\right)=\mathcal{E}(B)$. Since the ellipsoid $\mathcal{E}(B)$ is convex, it has to be complete Reinhardt.

Let $\left[a_{j k}\right]_{j, k=1, \ldots, n}$ be the matrix representation of the Hermitian scalar product $s\left(\widehat{h}_{B}\right)$ (recall $\left.s\left(\widehat{h}_{B}\right)(X, X)=\left(\widetilde{\mathbb{W}} \widehat{h}_{B}\right)^{2}(X)\right)$ in the canonical basis of $\mathbb{C}^{n}$, i.e. $s\left(\widehat{h}_{B}\right)(X, Y)=\sum_{j, k=1}^{n} a_{j k} X_{j} \bar{Y}_{k}, X, Y \in \mathbb{C}^{n}$. Then we have

$$
\mathcal{E}(B)=\left\{X \in \mathbb{C}^{n}: \sum_{j=1}^{n} a_{j j}\left|X_{j}\right|^{2}+2 \operatorname{Re} \sum_{j<k} a_{j k} X_{j} \bar{X}_{k}<1\right\},
$$

which is not invariant under $\Phi_{\Lambda}$ unless $a_{j k}=0$ for $j \neq k$.

From now on, we restrict ourselves to $\mathbb{C}^{2}$. However, some of the remarks can be easily transferred to the $n$-dimensional case.

Consider the mapping (cf. [Che-Kim 1], [Che-Kim 2])

$$
\Psi: \mathbb{C}^{2} \rightarrow \mathbb{R}_{+}^{2}, \quad \Psi(z, w):=\left(|z|^{2},|w|^{2}\right), \quad(z, w) \in \mathbb{C}^{2},
$$

which helps to develop the intuition how the ellipsoids $\mathcal{E}(B)$ look and will be useful in all computations performed in the sequel.

Notice that $\Psi$ transforms any bounded complete Reinhardt ellipsoid in $\mathbb{C}^{2}$ into a triangle

$$
T_{a, b}:=\left\{(u, v) \in \mathbb{R}_{+}^{2}: \frac{u}{a}+\frac{v}{b}<1\right\}
$$

for some $a, b>0$. In fact, the mapping $\Psi$ determines the one-to-one correspondence between bounded complete Reinhardt ellipsoids in $\mathbb{C}^{2}$ and triangles $T_{a, b} \subset \mathbb{R}_{+}^{2}$.

Let $\partial^{\circ} T_{a, b}$ denote the hypotenuse of the triangle $T_{a, b}$, i.e.

$$
\partial^{\circ} T_{a, b}:=\left\{(u, v) \in \bar{T}_{a, b}: \frac{u}{a}+\frac{v}{b}=1\right\} .
$$




\section{Lemma 4.}

(a) $T_{a, b} \subset T_{c, d}$ if and only if $a \leq c$ and $b \leq d$.

(b) If $B \subset \subset \mathbb{C}^{2}$ is a balanced Reinhardt domain, then $\Psi(\mathcal{E}(B))$ is a (unique) triangle $T_{a, b}$ of smallest area that contains $\Psi(B)$. Moreover, $h_{\mathcal{E}(B)}^{2}(X)=$ $\frac{\left|X_{1}\right|^{2}}{a}+\frac{\left|X_{2}\right|^{2}}{b}, X \in \mathbb{C}^{2}$.

(c) $\Psi\left(\mathcal{E}\left(r_{1} \Delta \times r_{2} \Delta\right)\right)=T_{2 r_{1}^{2}, 2 r_{2}^{2}}$ for $r_{1}, r_{2}>0$.

(d) $\mathcal{E}\left(\frac{\sqrt{3}}{2} \Delta \times \frac{1}{2} \Delta\right) \not \subset \mathcal{E}\left(\mathbb{B}_{\|\cdot\|}(0)\right)$.

Proof. (a) is trivial.

(b) Use Remark 3. Let $a, b>0$ be such that $\left(h_{\mathcal{E}(B)}\right)^{2}(X)=\frac{\left|X_{1}\right|^{2}}{a}+\frac{\left|X_{2}\right|^{2}}{b}$, $X \in \mathbb{C}^{2}$. We have $\operatorname{vol}(\mathcal{E}(B))=\beta a b$, where $\beta$ is the volume of the Euclidean unit ball in $\mathbb{C}^{2}$. Therefore $\operatorname{vol}(\Psi(\mathcal{E}(B)))=\operatorname{vol}\left(T_{a, b}\right)=\frac{1}{2} a b=\frac{1}{2 \beta} \operatorname{vol}(\mathcal{E}(B))$.

(c) Let $c, d>0$ be such that $T_{c, d}=\Psi\left(\mathcal{E}\left(r_{1} \Delta \times r_{2} \Delta\right)\right)$. Note that $\Psi\left(r_{1} \Delta \times r_{2} \Delta\right)=$ $\left[0, r_{1}^{2}\right] \times\left[0, r_{2}^{2}\right]$ and $\left(r_{1}^{2}, r_{2}^{2}\right) \in \partial^{\circ} T_{c, d}$, i.e. $\frac{r_{1}^{2}}{c}+\frac{r_{2}^{2}}{d}=1$. Finding $c, d$ boils down now to finding the minimum of the function

$$
V(c):=\operatorname{vol}\left(T_{c, d(c)}\right)=\frac{c^{2} r_{2}^{2}}{2\left(c-r_{1}^{2}\right)} .
$$

(d) It is a straightforward consequence of (a)-(c), namely we get

$$
\Psi\left(\mathcal{E}\left(\frac{\sqrt{3}}{2} \Delta \times \frac{1}{2} \Delta\right)\right)=T_{\frac{3}{2}, \frac{1}{2}}, \quad \Psi\left(\mathcal{E}\left(B_{\|\cdot\|}(0)\right)\right)=T_{1,1}
$$

Lemma 4 (d) shows that the inclusion $B_{1} \subset \subset B_{2}$ does not imply $\mathcal{E}\left(B_{1}\right) \subset \mathcal{E}\left(B_{2}\right)$, even for very regular domains $B_{1}, B_{2}$. It is where the idea of a counterexample comes from. For a large $R$ we are going to construct a domain $G \subset \mathbb{C}^{2}$ such that $(1, C) \in \overline{\mathbb{B}_{\alpha_{G}}(0)} \subset \bar{\Delta} \times 2 \bar{\Delta}$ for some $C=C(R)>0$ and at the same time $(0, R) \in \overline{\mathbb{B}_{\alpha_{G}}\left(z_{\nu}\right)} \subset \bar{\Delta} \times R \bar{\Delta}$ for $z_{\nu} \in G, z_{\nu} \rightarrow 0$. The mentioned $\mathbb{B}_{\alpha_{G}}$ will be Reinhardt. So $\Psi\left(\mathcal{E}\left(\mathbb{B}_{\alpha_{G}}(0)\right)\right)$ and $\Psi\left(\mathcal{E}\left(\mathbb{B}_{\alpha_{G}}\left(z_{\nu}\right)\right)\right)$ will be equal to, resp., $T_{a, b}$ and $T_{a_{\nu}, b_{\nu}}$ for some $a, b, a_{\nu}, b_{\nu}>0$ with $b_{\nu} \geq R^{2} \gg 1$ and desirably $a_{\nu}<a$. It means that even if $\mathbb{B}_{\alpha_{G}}(0) \subset \mathbb{B}_{\alpha_{G}}\left(z_{\nu}\right)$ for large $\nu$ (because of the possible upper semicontinuity of $\alpha_{G}$ ) we are able to arrange everything to end with $T_{a, b} \not \subset T_{a_{\nu}, b_{\nu}}$ (for sufficiently large $\nu$ ). Having in mind that the Wu metric at a point $z$ is simply a Minkowski functional of the ellipsoid $\mathcal{E}\left(\mathbb{B}_{\alpha_{G}}(z)\right)$ we conclude that $W \alpha_{G}$ is not upper semicontinuous at the point $0 \in G$.

Define a subharmonic function $u: \Delta \rightarrow(-\infty, 1]$

$$
u(z):=1+\sum_{j=4}^{\infty} \frac{1}{2^{j}} \max \left\{\log \frac{\left|2^{-j}-z\right|}{2},-2^{2 j}\right\}, \quad z \in \Delta,
$$

and put for $R>1$

$$
\begin{aligned}
G_{R} & :=\left\{(z, w) \in \Delta \times R \Delta: 2 R|w| e^{u(z)}<1\right\}, \\
\Omega_{R} & :=\left(\Delta \times \frac{1}{2 R} \Delta\right) \cup\left(\frac{1}{2} \Delta \times R \Delta\right), \\
\widehat{\Omega}_{R} & :=\text { the envelope of holomorphy of } \Omega_{R}, \\
C=C_{R} & :=\frac{1}{2 e R} .
\end{aligned}
$$


Let us also fix a holomorphically contractible family of pseudometrics $\left(\alpha_{D}\right)_{D}$ for $D \subset \mathbb{C}^{n}, n \geq 1$, satisfying the following condition for any domain $D \subset \mathbb{C}^{n}$ :

$$
A_{D}(a ; X) \leq \alpha_{D}(a ; X) \leq \kappa_{D}(a ; X), \quad a \in D, X \in \mathbb{C}^{n} .
$$

Clearly $\alpha_{D} \in \mathcal{M}(D)$.

We can precisely describe neither balls $\mathbb{B}_{\alpha_{G}}$ nor ellipsoids $\mathcal{E}\left(\mathbb{B}_{\alpha_{G}}\right)$. Nevertheless, the next proposition gives enough information to prove the main result (Proposition 6). We emphasize that $\mathbb{B}_{\alpha_{G_{R}}}(0) \subset(\Delta \times 2 \Delta)$ independently of the choice of the number $R$. The suitable $R$ will be fixed later in the proof of Proposition 6 .

Proposition 5. Let $R>1$ be a fixed number.

(a) $u(0)=1-\frac{3}{4} \log 2>0$ and $u(z)>0$ for $\frac{1}{4} \leq|z|<1$.

(b) $\Delta \times C \Delta \subset G_{R} \subset\left(\Delta \times \frac{1}{2 R} \Delta\right) \cup\left(\frac{1}{4} \Delta \times R \Delta\right) \subset \Omega_{R}$.

(c) $\mathcal{E}\left(\mathbb{B}_{\alpha_{G_{R}}}((z, 0))\right)$ is a complete Reinhardt domain for any $z \in \Delta$.

(d) $\mathbb{B}_{\alpha_{G_{R}}}(0) \subset(\Delta \times 2 \Delta) \cap \widehat{\Omega}_{R}$. Moreover, $(1, C) \in \partial \mathbb{B}_{\alpha_{G_{R}}}(0)$.

(e) $\mathbb{B}_{\alpha_{G_{R}}}\left(\left(2^{-\nu}, 0\right)\right) \subset \widehat{\Omega}_{R}$ and $(0, R) \in \partial \mathbb{B}_{\alpha_{G_{R}}}\left(\left(2^{-\nu}, 0\right)\right)$ for sufficiently large $\nu \in \mathbb{N}$.

Proof. (a) $u(0)=1+\sum_{j=4}^{\infty} \frac{\log 2^{-j-1}}{2^{j}}=1-\log 2 \sum_{j=4}^{\infty} \frac{j+1}{2^{j}}=1-\frac{3}{4} \log 2$. On the other hand, for $|z| \geq \frac{1}{4}, j \geq 3$, we have $\left|z-\frac{1}{2^{j}}\right| \geq \frac{1}{8}$ and $u(z) \geq 1+\sum_{j=4}^{\infty} \frac{\log 2^{-4}}{2^{j}}=$ $1-\frac{1}{2} \log 2>0$.

(b) If $|z|<1,|w|<C$, then $2 R|w| e^{u(z)}<1$ because $u(z)<1$. To prove the second inclusion, it is enough to show $|z| \geq \frac{1}{4} \Longrightarrow|w|<\frac{1}{2 R}$ for $(z, w) \in G_{R}$. But by (a) we have $1>2 R|w| e^{u(z)}>2 R|w|$.

(c) $\mathbb{B}_{\alpha_{G_{R}}}((z, 0))$ is a balanced domain; then it is invariant under the action of $\Phi_{(\lambda, \lambda)}, \lambda \in \bar{\Delta}$. On the other hand, the domain $G_{R}$ is invariant under $\Phi_{(1, \mu)}, \mu \in \bar{\Delta}$, and so is $\mathbb{B}_{\alpha_{G_{R}}}((z, 0))$. By Remark 3 the ellipsoid $\mathcal{E}\left(\mathbb{B}_{\alpha_{G_{R}}}((z, 0))\right)$ is complete Reinhardt.

(d) Let $v(z, w):=\frac{1}{2}\left(|w| e^{u(z)}+|z|\right),(z, w) \in G_{R}$. Notice that $v$ is a log-psh function, $v(z, w) \leq e\|(z, w)\|$, and moreover, $v(z, w) \leq \frac{1}{4 R}+\frac{1}{2}<1$ for all $(z, w) \in$ $G_{R}$. Hence, we obtain for $X=\left(X_{1}, X_{2}\right) \in \mathbb{C}^{2}$

$$
\alpha_{G_{R}}(0 ; X) \geq A_{G_{R}}(0 ; X) \geq \limsup _{0 \neq \lambda \rightarrow 0} \frac{v(\lambda X)}{|\lambda|}=\frac{1}{2}\left(\left|X_{2}\right| e^{u(0)}+\left|X_{1}\right|\right) .
$$

The domain $\widehat{\Omega}_{R}$ is a pseudoconvex Reinhardt domain, thus $\mathbb{B}_{A_{\widehat{\Omega}_{R}}}(0)=\widehat{\Omega}_{R}$ (cf. $\left[\right.$ Jar-Pfl 1]). Then, by (b) and condition (3), we get that $\mathbb{B}_{\alpha_{G_{R}}}(0) \subset \widehat{\Omega}_{R}$. Since $(1, C) \in \partial \widehat{\Omega}_{R}$, it is necessarily $\alpha_{G_{R}}(0 ;(1, C)) \geq 1$. In fact, the equality holds here. Indeed, take $\varphi(\lambda):=(\lambda, C \lambda), \lambda \in \Delta$. By (b) we have $\varphi(\Delta) \subset G_{R}$ and, consequently, $\alpha_{G_{R}}(0 ;(1, C)) \leq \kappa_{G_{R}}(0 ;(1, C)) \leq 1$.

(e) For natural numbers $\nu \geq 4$ define the biholomorphic mappings

$$
\Phi_{\nu}(z, w):=\left(\frac{z-2^{-\nu}}{1-2^{-\nu} z}, w\right), \quad(z, w) \in \Delta \times \mathbb{C},
$$

and notice that $\Phi_{\nu}\left(\frac{1}{2^{\nu}}, 0\right)=(0,0), \Phi_{\nu}\left(G_{R}\right) \subset \widehat{\Omega}_{R}, \nu \geq 4$. Indeed, in view of Proposition 5 (b), it is enough to verify by a direct computation that $|z|<\frac{1}{4} \Longrightarrow$ $\frac{\left|z-2^{-\nu}\right|}{\left|1-2^{-\nu} z\right|}<\frac{1}{2}$. Take $X=\left(X_{1}, X_{2}\right) \in \mathbb{B}_{\alpha_{G_{R}}}\left(\left(2^{-\nu}, 0\right)\right)$. Due to the contractibility of 
$\alpha$ we get

$$
\begin{aligned}
\alpha_{\widehat{\Omega}_{R}}\left((0,0) ;\left(\frac{1}{1-2^{-2 \nu}} X_{1}, X_{2}\right)\right)=\alpha_{\widehat{\Omega}_{R}}\left(\Phi_{\nu}\left(2^{-\nu}, 0\right) ;\right. & \left.\Phi_{\nu}^{\prime}\left(2^{-\nu}, 0\right) X\right) \\
& \leq \alpha_{G_{R}}\left(\left(2^{-\nu}, 0\right) ; X\right)<1
\end{aligned}
$$

The domain $\widehat{\Omega}_{R}$ is complete Reinhardt and so is the ball $\mathbb{B}_{\alpha_{\hat{\Omega}_{R}}}(0)$. Therefore $X \in \mathbb{B}_{\alpha_{\hat{\Omega}_{R}}}(0)$. In virtue of condition (3), we obtain

$$
\mathbb{B}_{\alpha_{G_{R}}}\left(\left(2^{-\nu}, 0\right)\right) \subset \mathbb{B}_{\alpha_{\widehat{\Omega}_{R}}}(0) \subset \mathbb{B}_{A_{\widehat{\Omega}_{R}}}(0)=\widehat{\Omega}_{R}
$$

The latter part of the statement can be obtained by repeating the argument from the proof of $(\mathrm{d})$ with the mappings $\varphi_{\nu}(\lambda):=\left(2^{-\nu}, R \lambda\right), \lambda \in \Delta$, for sufficiently large $\nu \in \mathbb{N}$.

For a number $R>1$ put

$$
A=A(R):=\frac{4-\frac{1}{2} C^{2}}{4-C^{2}}=\frac{32 e^{2} R^{2}-1}{32 e^{2} R^{2}-2} .
$$

The number $A$ is chosen in such a way that the points $\left(\frac{1}{2}, 4\right),\left(1, C^{2}\right),(A, 0)$ are collinear. In other words $\left(\frac{1}{2}, 4\right),\left(1, C^{2}\right) \in \partial^{\circ} T_{A, b_{0}}$ for some $b_{0}$ (precisely, $b_{0}=$ $\left.8-C^{2}\right)$.

\section{Proposition 6.}

(a) For any $R>1$ we have $(\sqrt{A}, 0) \in \mathcal{E}\left(\mathbb{B}_{\alpha_{G_{R}}}(0)\right)$.

(b) For any $R \geq 5$ there exists $\nu_{0}(R) \in \mathbb{N}$ such that $(\sqrt{A}, 0) \notin \mathcal{E}\left(\mathbb{B}_{\alpha_{G_{R}}}\left(\left(2^{-\nu}, 0\right)\right)\right)$ for $\nu \geq \nu_{0}(R)$.

Corollary 7. Let $R \geq 5$ be a fixed number.

(a) $\mathbb{W} \alpha_{G_{R}}$ is not upper semicontinuous at the point $(0 ;(1,0))$.

(b) If the family of pseudometrics $\left(\alpha_{D}\right)_{D}$ has the product property (1), then $\mathbb{W} \alpha_{G_{R} \times \Delta^{n-2}}(n \geq 2)$ is not upper semicontinuous at $(0 ;(1,0, \ldots, 0))$.

Proof of Corollary 7. (a) Take a number $\nu_{0}(R)$ such as in Proposition 6 (b). Since $\widetilde{\mathbb{W}} \alpha_{G_{R}}$ is the Minkowski functional of $\mathcal{E}\left(\mathbb{B}_{\alpha_{G_{R}}}\right.$ ) (cf. Remark $2(\mathrm{~b})$ ), then for $\nu \geq$ $\nu_{0}(R)$ we get

$$
\begin{aligned}
& \mathbb{W} \alpha_{G_{R}}(0 ;(1,0))=\sqrt{2} \widetilde{\mathbb{W}} \alpha_{G_{R}}(0 ;(1,0))<\frac{\sqrt{2}}{\sqrt{A}} \\
& \quad \leq \sqrt{2} \widetilde{\mathbb{W}} \alpha_{G_{R}}\left(\left(2^{-\nu}, 0\right) ;(1,0)\right)=\mathbb{W} \alpha_{G_{R}}\left(\left(2^{-\nu}, 0\right) ;(1,0)\right) .
\end{aligned}
$$

Conclude with $\nu \rightarrow \infty$.

(b) From Proposition 1 (d) we get

$$
\mathbb{W} \alpha_{G_{R} \times \Delta^{n-2}}((z, w) ;(1,0, \ldots, 0))=\mathbb{W} \alpha_{G_{R}}(z ;(1,0))
$$

for $(z, w) \in G_{R} \times \Delta^{n-2}$ and we apply (a). 
Proof of Proposition 6. Crucial for our consideration will be the geometry of the domain $\widehat{\Omega}_{R}$. We give here the precise description:

$$
\begin{gathered}
\log \left(\widehat{\Omega}_{R}\right)=\left\{(x, y) \in \mathbb{R}^{2}: x<0, y<\log R,\right. \\
\left.y<-\frac{\log \left(2 R^{2}\right)}{\log 2} x-\log (2 R)\right\}, \\
\widehat{\Omega}_{R}=\left\{(z, w) \in \mathbb{C}^{2}:|z|<1,|w|<R, 2 R|z|^{\frac{\log \left(2 R^{2}\right)}{\log 2}}|w|<1\right\}, \\
\Psi\left(\widehat{\Omega}_{R}\right)=\left\{(u, v) \in \mathbb{R}_{+}^{2}: u<1, v<R^{2}, 4 R^{2} u^{\frac{\log \left(2 R^{2}\right)}{\log 2}} v<1\right\} .
\end{gathered}
$$

Notice a very similar "shape" of $\Psi\left(\widehat{\Omega}_{R}\right)$ and $\left|\widehat{\Omega}_{R}\right|:=\left\{(|z|,|w|) \in \mathbb{R}_{+}^{2}:(z, w) \in \widehat{\Omega}_{R}\right\}$. We will especially make use of the convexity of the function $v(u)=\left(4 R^{2} u^{\frac{\log \left(2 R^{2}\right)}{\log 2}}\right)^{-1}$ for $\frac{1}{4} \leq u \leq 1$.

Recall that by Proposition 5 (c) the ellipsoids of our interest are bounded complete Reinhardt domains. Thus, we are allowed to use triangles $T_{c, d}$ for proving or disproving the minimality of those ellipsoids. To prove both (a) and (b) we are going to proceed in the following way: we fix $T$ being a triangle corresponding to the ellipsoid $\mathcal{E}\left(\mathbb{B}_{\alpha_{G}}\right)$ and assume for a contradiction that the claim does not hold. Then we manipulate the triangle rotating its hypotenuse slightly around a certain fixed point from $\partial^{\circ} T$. Performing the rotation, we are able to decrease the area of the triangle and provide at the same time (with the use of Proposition 5 (d),(e)) that it still contains $\Psi\left(\mathbb{B}_{\alpha_{G}}\right)$. It obviously contradicts the minimality of the initial triangle $T$.

Recall that the number $A=A(R)>1$ (cf. (4)) was chosen such that $\left(\frac{1}{2}, 4\right),\left(1, C^{2}\right)$ $\in \partial^{\circ} T_{A, b_{0}}$.

Proof of Proposition 6 (a). Let $a, b>0$ be such that

$$
T_{a, b}=\Psi\left(\mathcal{E}\left(\mathbb{B}_{\alpha_{G_{R}}}(0)\right)\right) .
$$

Suppose that the statement (a) does not hold, i.e. $a \leq A$.

Consider the following condition:

$$
\text { there exist } v_{1}>0, u_{2}>\frac{2}{3}, v_{2}>0 \text { such that }
$$

$$
\overline{\Psi\left(\widehat{\Omega}_{R}\right)} \cap([0,1] \times[0,4]) \cap \partial^{\circ} T_{a, b}=\left[\left(u_{2}, v_{2}\right),\left(1, v_{1}\right)\right] .
$$

First we show that condition (7) is sufficient to establish a contradiction, then we prove (7).

Note that condition (7) remains true if we substitute $(a, b)$ by $(\tilde{a}, \tilde{b})$ from a small neigborhood of $(a, b)$. We will rotate the hypotenuse of the triangle $T_{a, b}$ around the point $\left(u_{2}, v_{2}\right)$. Define the family

$$
\mathfrak{T}\left(u_{2}, v_{2}\right):=\left\{T_{c, d(c)}:\left[0, u_{2}\right] \times\left[0, v_{2}\right] \subset \bar{T}_{c, d(c)},\left(u_{2}, v_{2}\right) \in \partial^{\circ} T_{c, d(c)}\right\},
$$

where $d(c)$ is a function of $c$. Certainly, $T_{a, b} \in \mathfrak{T}\left(u_{2}, v_{2}\right)$.

By Lemma 4 (c), $T_{2 u_{2}, 2 v_{2}}$ is a triangle of minimal area in $\mathfrak{T}\left(u_{2}, v_{2}\right)$. Moreover, the volume function $c \mapsto \operatorname{vol}\left(T_{c, d(c)}\right)$ is decreasing for $c \in\left(a, 2 u_{2}\right)$. (Note that $a \leq A<\frac{4}{3}<2 u_{2}$.) On the other hand, by Proposition 5 (d) and properties (5), 
(7), we see that for sufficiently small $\varepsilon>0$

$$
\begin{aligned}
\Psi\left(\mathbb{B}_{\alpha_{G_{R}}}(0)\right) \subset([0,1] \times[0,4]) \cap \Psi\left(\widehat{\Omega}_{R}\right) & \cap T_{a, b} \\
& \subset([0,1] \times[0,4]) \cap \Psi\left(\widehat{\Omega}_{R}\right) \cap T_{a+\varepsilon, d(a+\varepsilon)} .
\end{aligned}
$$

This contradicts the minimality of the triangle $T_{a, b}$ (condition (6)).

To prove $(7)$, we show that there exist $u_{0} \in\left(\frac{2}{3}, 1\right], v_{0}>0$ such that

$$
\overline{\Psi\left(\widehat{\Omega}_{R}\right)} \cap([0,1] \times[0,4]) \cap \partial^{\circ} T_{A, b_{0}}=\left[\left(u_{0}, v_{0}\right),\left(1, C^{2}\right)\right] .
$$

This can be seen by taking a look at the logarithmic image of $\widehat{\Omega}_{R}$ (cf. (5)) and verifying that e.g. $\left(\frac{1}{2} \log \frac{2}{3}, 0\right) \notin \overline{\log \left(\widehat{\Omega}_{R}\right)}$. Thus, $\left(\frac{2}{3}, 1\right) \in T_{A, b_{0}} \backslash \overline{\Psi\left(\widehat{\Omega}_{R}\right)}$, which, together with $\left(\frac{1}{2}, 4\right) \in \bar{T}_{A, b_{0}} \backslash \overline{\Psi\left(\widehat{\Omega}_{R}\right)}$, means that

$$
\left[\left(\frac{1}{2}, 4\right),\left(\frac{2}{3}, 1\right)\right] \subset \bar{T}_{A, b_{0}} \backslash \overline{\Psi\left(\widehat{\Omega}_{R}\right)} .
$$

This implies (9) because $\left[\left(\frac{1}{2}, 4\right),\left(1, C^{2}\right)\right] \in \partial^{0} T_{A, b_{0}}$.

Since we assumed that $a \leq A$ and we have $(1, C) \in \partial \mathbb{B}_{\alpha_{G_{R}}}(0)$ (cf. Proposition $5(\mathrm{~d}))$, the slope of $\partial^{\circ} T_{a, b}$ is steeper than $\partial^{\circ} T_{A, b_{0}}$ and $\left(1, C^{2}\right) \in \bar{T}_{a, b}$. This fact and conditions (5), (9) imply (7).

Proof of Proposition 6 (b). Let $a_{\nu}, b_{\nu}>0$ be such that

$$
T_{a_{\nu}, b_{\nu}}=\Psi\left(\mathcal{E}\left(\mathbb{B}_{\alpha_{G_{R}}}\left(\left(2^{-\nu}, 0\right)\right)\right)\right), \quad \nu \geq 1 .
$$

We show that for $R \geq 5$

$$
\left[\left(\frac{1}{3}, \frac{R^{2}}{2}\right),\left(1, \frac{1}{4 R^{2}}\right)\right) \subset T_{A, R^{2}} \backslash \overline{\Psi\left(\widehat{\Omega}_{R}\right)}
$$

First, let us check that $\left(\frac{1}{3}, \frac{R^{2}}{2}\right) \in T_{A, R^{2}} \backslash \overline{\Psi\left(\widehat{\Omega}_{R}\right)}$. It is equivalent to

$$
\left\{\begin{array}{r}
\frac{1}{3 A}+\frac{1}{2}<1, \\
2 R^{4}>3^{\frac{\log \left(2 R^{2}\right)}{\log 2}},
\end{array}\right.
$$

which holds for $R \geq 2$. Obviously, $\left(1, \frac{1}{4 R^{2}}\right) \in \partial \Psi\left(\widehat{\Omega}_{R}\right)$. On the other hand, the condition $\left(1, \frac{1}{4 R^{2}}\right) \in T_{A, R^{2}}$ reduces to the inequality

$$
\frac{1}{A}+\frac{1}{4 R^{4}}<1 \Longleftrightarrow-4 R^{4}+32 e R^{2}-1<0,
$$

which holds for $R \geq 5$. This proves (11).

Fix an $R$ such that condition (11) is satisfied and take a $\nu_{0}(R)$ so large that Proposition 5 (e) holds for $\nu \geq \nu_{0}(R)$.

Suppose that for a certain fixed $\nu \geq \nu_{0}(R)$ the claim of (b) does not hold. That is equivalent to $a_{\nu}>A$. We have necessarily $b_{\nu} \geq R^{2}$ because $(0, R) \in$ $\partial \mathbb{B}_{\alpha_{G_{R}}}\left(\left(2^{-\nu}, 0\right)\right)$ (cf. Proposition $\left.5(\mathrm{e})\right)$. Thus, by Lemma 4 (a) we have

$$
T_{A, R^{2}} \subset T_{a_{\nu}, b_{\nu}} \text {. }
$$

We claim that there exist some $0<u_{3}<\frac{1}{3}, v_{3}>0$ such that

$$
\left(u_{3}, v_{3}\right) \in \overline{\Psi\left(\widehat{\Omega}_{R}\right)} \cap \partial^{\circ} T_{a_{\nu}, b_{\nu}} \subset\left[\left(0, b_{\nu}\right),\left(u_{3}, v_{3}\right)\right] .
$$


Indeed, it suffices to see that e.g.

$$
\left[\left(\frac{1}{3}, \frac{R^{2}}{2}\right),\left(1, \frac{1}{4 R^{2}}\right)\right) \subset T_{a_{\nu}, b_{\nu}} \backslash \overline{\Psi\left(\widehat{\Omega}_{R}\right)}
$$

which follows by (11) and (12).

Now, take the family $\mathfrak{T}\left(u_{3}, v_{3}\right)$ defined as in (8). Obviously, $T_{a_{\nu}, b_{\nu}} \in \mathfrak{T}\left(u_{3}, v_{3}\right)$. We repeat (almost literally) the argument from the proof of (a).

By Lemma 4 (c), $T_{2 u_{3}, 2 v_{3}}$ is a triangle of minimal area in $\mathfrak{T}\left(u_{3}, v_{3}\right)$. Moreover, the volume function $c \mapsto \operatorname{vol}\left(T_{c, d(c)}\right)$ is increasing for $c \in\left(2 u_{3}, a_{\nu}\right)$. (Recall that $2 u_{3}<A<a_{\nu}$.) On the other hand, by Proposition 5 (e) and properties (5), (13), we obtain for sufficiently small $\varepsilon>0$

$$
\Psi\left(\mathbb{B}_{\alpha_{G_{R}}}\left(2^{-\nu}, 0\right)\right) \subset \Psi\left(\widehat{\Omega}_{R}\right) \cap T_{a_{\nu}, b_{\nu}} \subset \Psi\left(\widehat{\Omega}_{R}\right) \cap T_{a_{\nu}-\varepsilon, d\left(a_{\nu}-\varepsilon\right)} .
$$

This contradicts the minimality of $T_{a_{\nu}, b_{\nu}}$ (condition (10)).

\section{ACKNOWLEDGMENTS}

The author would like to thank the referee, and his professors and colleagues for pointing out numerous mistakes in the original version of the paper.

\section{REFERENCES}

[Che-Kim 1] C. K. Cheung, K. T. Kim, Analysis of the Wu metric. I: The case of convex Thullen domains, Trans. Amer. Math. Soc. 348 (1996), 1421-1457. MR1357392 (96i:32026)

[Che-Kim 2] C. K. Cheung, K. T. Kim, Analysis of the Wu metric. II: The case of non-convex Thullen domains, Proc. Amer. Math. Soc. 125 (1997), 1131-1142. MR1363414 (97f:32029)

[Jar-Pfl 1] M. Jarnicki, P. Pflug, Invariant Distances and Metrics in Complex Analysis, de Gruyter Exp. Math. 9, de Gruyter, Berlin, 1993. MR1242120 (94k:32039)

[Jar-Pfl 2] M. Jarnicki, P. Pflug, Invariant distances and metrics in complex analysisrevisited, Dissertationes Math. 430 (2003). MR2167637 (2006h:32010)

[Jar-Pfl 3] M. Jarnicki, P. Pflug, On the upper semicontinuity of the Wu metric, Proc. Amer. Math. Soc. 133 (2005), 239-244. MR2086216 (2005g:32012)

[Juc] P. Jucha, The Wu metric in elementary Reinhardt domains, Univ. Iagel. Acta Math. 38 (2000), 169-184. MR1962715 (2003m:32008)

[Wu 1] H. Wu, Unpublished notes.

[Wu 2] $\mathrm{H}$. Wu, Old and new invariant metrics on complex manifolds, in: Several Complex Variables, J. E. Fornæss (ed.), Math. Notes 38, Princeton Univ. Press, 1993, 640682. MR1207887 (94a:32038)

Institute of Mathematics, Jagiellonian University, Reymonta 4, 30-059 Kraków, POLAND

E-mail address: Piotr.Jucha@im.uj.edu.pl 